Bundesgesundheitsbl - Gesundheitsforsch Gesundheitsschutz 2004 · 47:1189-1195 DOI 10.1007/s00103-004-0950-0 ๑) Springer Medizin Verlag 2004

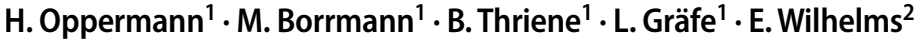 \\ C. Herrmann ${ }^{2} \cdot$ H. Weise ${ }^{3} \cdot$ E. Hennig ${ }^{3} \cdot$ H. Claus ${ }^{4}$ \\ ${ }^{1}$ Landesamt für Verbraucherschutz Sachsen-Anhalt \\ ${ }^{2}$ Fachbereich Gesundheit/Veterinärwesen der Stadt Halle (Gesundheitsamt) \\ ${ }^{3}$ Gesundheits- und Veterinäramt Magdeburg . ${ }^{4}$ Robert Koch-Institut, Berlin
}

\title{
Erfahrungen und Schwierigkeiten bei der Implementierung von Impfregistern in Sachsen-Anhalt
}

Bevölkerung angestrebt wird. In der Folge wurde ein Arbeitskreis „Impfen“ gegründet, der sich aus Vertretern aller mit dem Impfen befassten Institutionen und Verbänden zusammensetzt und der durch Kooperation und Vernetzung eine Verbesserung der Impfsituation erreichen möchte [2]. Die Impfmeldepflicht war von Anfang an ein wichtiges Thema des Arbeitskreises. Obgleich versucht wurde, die niedergelassenen Ärzte durch Informationsbeiträge und Aufrufe in der Zeitschrift „Pro“ der Kassenärztlichen Vereinigung [3, 4, 5,] zur Meldung zu motivieren, stellte sich bald heraus, dass die Umsetzung in den 21 Landkreisen und 3 kreisfreien Städten Schwierigkeiten bereitete. Viele Ärzte ignorierten entweder die Meldepflicht oder meldeten nichtnamentlich in einer Form, die sich für das Anlegen eines Impfregisters als ungeeignet erwies. Nur aus wenigen Gesundheitsämtern wurde über ein gutes Meldeverhalten niedergelassener Ärzte berichtet. Diese Gesundheitsämter hatten zumeist über eigene Aktivitäten vor allem die Kinderärzte ihrer Region zum Melden motiviert. Unsicherheiten gab es in der Regel bei der Bearbeitung und Analyse der Daten in einem elektronischen Impfregister.

Im Jahr 2002 gelangte der Arbeitskreis „Impfen“ zu der Auffassung, dass die Umsetzbarkeit der Impfmeldepflicht in Sachsen-Anhalt, der Aufbau eines elektronischen Impfregisters in den Gesundheitsämtern sowie Aufwand und Nutzen einer darauf aufbauenden Impferinnerung bei fehlenden Impfungen mit einem Pilotprojekt analysiert und bewertet werden sollte. Voraussetzung für die namentliche Meldung der Impfungen durch den Arzt ist die schriftliche Zustimmung der Eltern. Um die Einstellung der Eltern zur namentlichen Meldung bzw. Hinderungsgründe einschätzen zu können, wurde eine Befragung junger Mütter durchgeführt. Zusätzlich wurden niedergelassene Ärzte zur Impfmeldepflicht befragt. Deren Einfluss auf die Impfakzeptanz von Eltern und $\mathrm{Pa}$ tienten ist bekannt [6] und lässt einen nicht unerheblichen Einfluss auf die Einstellung der Eltern zur Impfmeldepflicht vermuten. Es ist daher wichtig, Vorbehalte der Ärzte gegenüber der Impfmeldepflicht zu kennen, um sie sachlich zu entkräften und eine positive Haltung zu fördern. Nur dann wird der Arzt in der Lage sein, die namentliche Impfmeldepflicht den Eltern gegenüber zu vertreten und deren Zustimmung zu erreichen. Eine Befragung aller 24 Gesundheitsämter in Sachsen-Anhalt sollte der besseren Einschätzung und Vergleichbarkeit der Umsetzung der Meldepflicht nach $\$ 4$ GDG LSA dienen, aber auch Erfahrungen mit dem Impfregister aus der DDR-Zeit herausarbeiten. Die Befragungen junger Mütter und niedergelassener Ärzte wurden in der Stadt Halle/Saale und in der Landeshauptstadt Magdeburg durchgeführt. In den Gesundheitsämtern dieser Städte ziel zum Ausdruck, nach dem eine altersgerechte Durchimpfung von über 90\% der 
Tabelle 1

Ergebnisse der Befragung junger Mütter zur Impfmeldepflicht

\begin{tabular}{|c|c|c|c|}
\hline Fragebogenangabe & Magdeburg & Halle & Gesamt \\
\hline Impfmeldepflicht ist bekannt & $138 / 612(22,5 \%)^{a}$ & $163 / 541(30,1 \%)^{\mathrm{a}}$ & $301 / 1153(26,1 \%)^{a}$ \\
\hline \multicolumn{4}{|l|}{ Information durch: } \\
\hline • Kinderarzt & $99 / 129(76,7 \%)$ & $116 / 141(82,3 \%)$ & $215 / 270(79,6 \%)$ \\
\hline • Hausarzt & $2 / 129(1,5 \%)$ & $0 / 141(0 \%)$ & $2 / 270(0,7 \%)$ \\
\hline -Andere & $28 / 129(21,7 \%)$ & $25 / 141(17,7 \%)$ & $53 / 270(19,6 \%)$ \\
\hline $\begin{array}{l}\text { Zustimmung zur nament- } \\
\text { lichen Impfmeldepflicht }\end{array}$ & $489 / 610(80,2 \%)$ & $410 / 539(76,1 \%)$ & $899 / 1149(78,2 \%)$ \\
\hline $\begin{array}{l}\text { Zustimmung zur } \\
\text { Impferinnerung durch das } \\
\text { Gesundheitsamt }\end{array}$ & $513 / 610(84,1 \%)$ & $450 / 539(83,5 \%)$ & $963 / 1149(83,8 \%)$ \\
\hline $\begin{array}{l}\text { Positive bis sehr positive } \\
\text { Einstellung zu Impfungen } \\
\text { allgemein }\end{array}$ & $602 / 612(98,4 \%)^{a}$ & $510 / 539(94,6 \%)^{a}$ & $1112 / 1151(96,6 \%)^{\mathrm{a}}$ \\
\hline $\begin{array}{l}\text { Einschätzung, dass Kinder- } \\
\text { oder Hausarzt die nament- } \\
\text { liche Impfmeldepflicht } \\
\text { befürwortet }\end{array}$ & $269 / 610(44,1 \%)$ & $246 / 539(45,6 \%)$ & $515 / 1149(44,8 \%)$ \\
\hline $\begin{array}{l}\text { Einschätzung, dass Kinder- } \\
\text { oder Hausarzt die Bedenken } \\
\text { der Eltern bezüglich der } \\
\text { Impfmeldepflicht ausräumen } \\
\text { könnte }\end{array}$ & $24 / 116(20,7 \%)$ & $27 / 125(21,6 \%)$ & $51 / 241(21,2 \%)$ \\
\hline \multicolumn{4}{|c|}{ Gründe für die Ablehnung der Impfmeldepflicht: } \\
\hline - Datenschutz & $107 / 121(88,4 \%)$ & $121 / 129(93,8 \%)$ & $228 / 250(91,2 \%)$ \\
\hline • Keine Notwendigkeit & $24 / 121(19,8 \%)$ & $35 / 129(27,1 \%)$ & $59 / 250(23,6 \%)$ \\
\hline • Sonstige Gründe & $18 / 121(14,9 \%)$ & $16 / 129(12,4 \%)$ & $34 / 250(13,6 \%)$ \\
\hline
\end{tabular}

a Ortsunterschiede zwischen Magdeburg und Halle signifikant (Impfmeldepflicht bekannt: $O R=0,67$; 95\%-KI: 0,5-0,9; positive bis sehr positive Einstellung zum Impfen:OR=3,4; 95\%-KI: 1,6-7,1).

Tabelle 2

Einflussfaktoren auf die Zustimmung zur Impfmeldepflicht

\begin{tabular}{|c|c|c|}
\hline Einflussfaktoren & Odds Ratio & 95\%-Konfidenzintervall \\
\hline Impfmeldepflicht bekannt & 1,3 & $0,9-1,8$ \\
\hline Impfmeldepflicht durch Kinderarzt bekannt & 1,1 & $0,8-1,6$ \\
\hline Positive oder sehr positive Einstellung zum Impfen & 12,5 & $6,4-24,3$ \\
\hline $\begin{array}{l}\text { Einschätzung, dass Kinderarzt die namentliche } \\
\text { Impfmeldepflicht befürwortet }\end{array}$ & 8,1 & $5,5-11,9$ \\
\hline
\end{tabular}

sollte anschließend mit dem Aufbau eines elektronischen Impfregisters begonnen werden. $\mathrm{Zu}$ diesem Zweck musste eine geeignete Software erarbeitet, in den Gesundheitsämtern installiert und dafür gesorgt werden, dass möglichst viele Meldungen in guter Qualität eingehen. Über erste Erkenntnisse und Erfahrungen im Zusammenhang mit diesem Projekt wird nachfolgend berichtet.

\section{Methoden}

\section{Befragung junger Mütter zur Akzeptanz der Impfmeldepflicht}

Mütter in Magdeburg und in Halle, die im Jahr 2002 ein Kind geboren hatten, wurden anonym mittels standardisiertem Fragebogen befragt. Die Befragung erfolgte postalisch nach Bereitstellung der Anschriften durch die Einwohnermeldeämter. In einem Anschreiben wurden den Müttern das Anliegen der Impfmeldepflicht und der Sinn der Befragung erläutert. Der Fragebogen bestand aus 7 Fragen. 4 Fragen galten dem Wissen sowie der Einstellung zur Impfmeldepflicht, zur Impferinnerung durch das Gesundheitsamt und zu Impfungen allgemein. Eine weitere Frage sollte klären, wie die Mütter die Einstellung ihres Kinder- bzw. Hausarztes zur Impfmeldepflicht beurteilen. In 2 Fragen wurden diejenigen Mütter, die eine namentliche Impfmeldepflicht ablehnten, nach den diesbezüglichen Gründen sowie danach, ob ihr Arzt ihre Bedenken hinsichtlich der Meldepflicht ausräumen könnte, befragt.

Die Befragung erfolgte im Dezember 2003. Es wurden 1200 Mütter in Halle und 1200 Mütter in Magdeburg angeschrieben. Nach 14 Tagen fand eine einmalige schriftliche Erinnerungsaktion statt. Zwischen der Bereitstellung der Adressen durch die Einwohnermeldeämter und der Befragung lagen etwa 6 Monate. Weil vermutlich eine nicht unerhebliche Zahl der Mütter in der Zwischenzeit umgezogen war [7], wurden von der Bruttostichprobe $10 \%$ abgezogen und diese Zahlen (je 1080 Mütter in Magdeburg und Halle) für die Berechnung der Response zugrunde gelegt.

\section{Befragung niedergelassener Kinder- und Hausärzte zur Impfmeldepflicht}

In Magdeburg gibt es 29 niedergelassene Kinderärzte und 144 Hausärzte. In Halle gibt es 32 Kinderärzte und 161 Hausärzte. Alle Ärzte wurden im Februar 2004 angeschrieben und gebeten, an der standardisierten anonymen Befragung zur Impfmeldepflicht teilzunehmen. Die Fragenkomplexe betrafen:

- den Bekanntheitsgrad der Impfmeldepflicht unter den Ärzten,

- die Einschätzung der Umsetzung der Impfmeldepflicht bei Berufskollegen,

- mögliche Hinderungsgründe für die Umsetzung der Impfmeldepflicht,

- Vorteile der Impfmeldepflicht,

- Einstellung zur Impferinnerung durch das Gesundheitsamt. 
Ärzte, die nach 14 Tagen noch keine Antwort übermittelt hatten, wurden einmal an die Befragung erinnert und gebeten, den Fragebogen doch noch zurückzuschicken.

\section{Befragung der Gesundheitsämter zur aktuellen Umsetzung der Impfmeldepflicht}

In Sachsen Anhalt gibt es 24 Gesundheitsämter in 21 Landkreisen und 3 kreisfreien Städten. Alle Gesundheitsämter wurden im Februar 2004 angeschrieben und um Teilnahme an der Befragung gebeten. Wesentliche Aspekte der Befragung waren

- Existenz und Nutzung des alten Impfregisters aus der DDR-Zeit,

- Einschätzung der Akzeptanz der Impfmeldepflicht durch die niedergelassene Ärzteschaft,

- Umgang der Gesundheitsämter mit den eingehenden Meldungen,

- Analyse möglicher Ursachen für die mangelnde Bereitschaft zur Impfmeldung.

\section{Datenauswertung und statistische Verfahren}

In EpiInfo 2002 wurden Eingabemasken für die standardisierten Fragebögen entworfen und die erhobenen Daten eingegeben. Die statistische Analyse erfolgte mit der Statistiksoftware SPSS für Windows (Version 12.0). Neben deskriptiven Größen wurden für die Beurteilung der Signifikanz von Unterschieden bei der Beantwortung der einzelnen Fragen jeweils Odds Ratios (OR) und 95\%-Konfidenzintervalle ( $95 \%$-KI) berechnet.

\section{Ergebnisse}

\section{Befragung junger Mütter zur Akzeptanz der Impfmeldepflicht}

Insgesamt antworteten 1163 von 216o Müttern der bereinigten Stichprobe auf die Befragung. Das entsprach einer Response von 53,8\%. In Magdeburg war die Response mit $57,5 \%$ (621) etwas höher als in Halle mit 50,2\% (542). Die Ergebnisse der Befragung sind in - Tabelle 1 dargestellt. Sie zeigen, dass nur einem kleinen Teil der Mütter die Impfmeldepflicht bekannt war. In

Bundesgesundheitsbl - Gesundheitsforsch -Gesundheitsschutz 2004 • 47:1189-1195

DOI 10.1007/s00103-004-0950-0

(c) Springer Medizin Verlag 2004

H. Oppermann · M. Borrmann - B. Thriene - L. Gräfe - E. Wilhelms · C. Herrmann

H. Weise $\cdot$ E. Hennig $\cdot$ H. Claus

\section{Erfahrungen und Schwierigkeiten bei der Implementierung von Impfregistern in Sachsen-Anhalt}

\section{Zusammenfassung}

In Sachsen-Anhalt sind Impfungen bei Kindern bis zum vollendeten 7. Lebensjahr an das Gesundheitsamt namentlich zu melden. Ziel der Meldepflicht ist die Verbesserung und Stabilisierung der altersgerechten Durchimpfung. Bei der Umsetzung der Meldepflicht gibt es Schwierigkeiten, die mit einem Modellprojekt in den Gesundheitsämtern Magdeburg und Halle analysiert und behoben werden sollen. Entscheidend für die Umsetzung der Meldepflicht sind das Einverständnis der Eltern, die Bereitschaft der impfenden Ärzte und das Vorliegen der personellen und technischen Möglichkeiten im Gesundheitsamt. Eine Befragung niedergelassener Kinder- und Hausärzte ergab, dass Vorbehalte gegen die Meldepflicht bezüglich des Datenschutzes und der rechtlichen Absicherung weit verbreitet sind. Die Hausärzte glauben außerdem, dass die Eltern mit ei- ner namentlichen Meldung nicht einverstanden sind. Dass dies nicht der Fall ist, ergab eine Befragung junger Mütter. Entscheidend für die Zustimmung junger Mütter zur Meldepflicht ist, dass der impfende Arzt ebenfalls eine positive Haltung einnimmt. Um die Impfmeldepflicht umzusetzen, ist es daher zwingend notwendig, die diesbezüglichen Bedenken der Ärzte auszuräumen und den unmittelbaren Nutzen der Meldungen in der täglichen Praxis erlebbar zu machen. Dies stellt hohe Anforderungen an das Gesundheitsamt, das ein Impfregister führen, die Kommunikation mit den Ärzten aufrechterhalten und die Eltern an fehlende Impfungen ihrer Kinder erinnern sollte.

\section{Schlüsselwörter}

Impfmeldepflicht · Impfregister · Junge Mütter · Impfende Ärzte · Gesundheitsamt

\section{Experiences and difficulties in implementing immunization registries in Saxony-Anhalt}

\section{Abstract}

In the German Federal State of Saxony-Anhalt, the Health Department must be notified of vaccines administered to children $<7$ years of age including their names. The goal of this mandatory notification is to improve and stabilize the age-appropriate vaccination coverage. However, difficulties have been encountered in implementing mandatory notification. Therefore, the Health Departments of Magdeburg and Halle have launched a model project focusing on analysing and eliminating these problems. Mandatory notification requires parents' approval, endorsement of vaccinators, and availability of human and technical resources in the Health Department. An enquiry among paediatricians and family doctors in private practice revealed widespread reservations about mandatory notification because of privacy issues related to data and legal protection. Furthermore, family doctors believed that parents disapproved of notification by name. However, a survey among young mothers revealed this not to be the case. Winning young mothers' approval depends largely on the positive attitude of the vaccinators. To implement the mandatory notification of vaccinations to the Health Department, it is necessary to dispel physicians' concerns and to inform young parents about the practical benefits. However, this places high demands on the Health Departments for maintaining immunization registries, communicating with physicians and notifying parents when immunisations are due or late.

\section{Keywords}

Obligation to notify the Health Department of vaccinations - Immunisation registries . Young mothers - Vaccinators . Health Department 
Tabelle 3

Ergebnisse der Befragung niedergelassener Ärzte zur Impfmeldepflicht

\begin{tabular}{|c|c|c|c|c|c|c|}
\hline \multirow[t]{2}{*}{ Fragebogenangabe } & \multicolumn{3}{|c|}{$\begin{array}{l}\text { Befragung niedergelassener Kinderärzte zur } \\
\text { Impfmeldepflicht }\end{array}$} & \multicolumn{3}{|c|}{$\begin{array}{l}\text { Befragung niedergelassener Hausärzte, } \\
\text { die in ihrer Praxis Kinder bis zum vollendeten } \\
\text { 7. Lebensjahr impfen }\end{array}$} \\
\hline & $\begin{array}{l}\text { Magdeburg } \\
\text { Antworten } \\
\text { mit ja }\end{array}$ & $\begin{array}{l}\text { Halle } \\
\text { Antworten } \\
\text { mit ja }\end{array}$ & Gesamt & $\begin{array}{l}\text { Magdeburg } \\
\text { Antworten } \\
\text { mit ja }\end{array}$ & $\begin{array}{l}\text { Halle } \\
\text { Antworten } \\
\text { mit ja }\end{array}$ & Gesamt \\
\hline Impfmeldepflicht ist bekannt & $19 / 21(90,5 \%)$ & $25 / 29(86,2 \%)$ & $44 / 50(88,0 \%)^{a}$ & $27 / 38(71,0 \%)$ & $27 / 45(60,0 \%)$ & $54 / 83(65,0 \%)^{a}$ \\
\hline \multicolumn{7}{|c|}{ Impfmeldepflicht ausreichend abgesichert? } \\
\hline • Rechtlich & $4 / 20(20,0 \%)$ & $9 / 29(31,0 \%)$ & $13 / 49(26,5 \%)$ & $16 / 38(42,1 \%)$ & $16 / 45(35,5 \%)$ & $32 / 83(38,5 \%)$ \\
\hline - Datenschutzrechtlich & $5 / 20(25,0 \%)$ & $10 / 29(34,5 \%)$ & $15 / 49(30,6 \%)$ & $16 / 38(42,1 \%)$ & $17 / 45(37,8 \%)$ & $33 / 83(39,7 \%)$ \\
\hline $\begin{array}{l}\text { Verursacht sie zu hohen Zeitaufwand } \\
\text { in der Praxis? }\end{array}$ & $8 / 20(40,0 \%)$ & $7 / 29(24,1 \%)$ & $15 / 49(30,6 \%)$ & $19 / 38(50,0 \%)$ & $16 / 45(35,5 \%)$ & $35 / 83(42,2 \%)$ \\
\hline $\begin{array}{l}\text { Sind die Eltern mit der namentlichen } \\
\text { Impfmeldepflicht einverstanden? }\end{array}$ & $12 / 18(66,7 \%)$ & $23 / 29(79,3 \%)$ & $35 / 47(74,5 \%)^{a}$ & $18 / 36(50,0 \%)$ & $15 / 42(35,7 \%)$ & $33 / 78(42,3 \%)^{\mathrm{a}}$ \\
\hline \multicolumn{7}{|l|}{ Vorteile aus der Impfmeldepflicht? } \\
\hline •Erkennen von Impflücken & $15 / 20(75,0 \%)$ & $27 / 29(93,1 \%)$ & $42 / 49(85,7 \%)$ & $34 / 38(89,6 \%)$ & $45 / 46(97,8 \%)$ & $79 / 84(94,0 \%)$ \\
\hline - Erfassen der Kinder, die keinem & $14 / 20(70,0 \%)$ & $20 / 29(69,0 \%)$ & $34 / 49(69,4 \%)$ & $31 / 38(81,6 \%)$ & $39 / 46(84,8 \%)$ & $70 / 84(83,3 \%)$ \\
\hline Arzt vorgestellt werden & & & & & & $68 / 83(81,9 \%)$ \\
\hline $\begin{array}{l}\text { - Aufforderung an die Eltern dieser } \\
\text { Kinder, sie einem Arzt vorzustellen }\end{array}$ & $13 / 18(72,2 \%)$ & $21 / 29(72,4 \%)$ & $34 / 47(72,3 \%)$ & $25 / 38(65,8 \%)$ & $43 / 45(95,5 \%)$ & \\
\hline $\begin{array}{l}\text { Sollte die Impfmeldepflicht mit einer } \\
\text { Impferinnerung verbunden sein? }\end{array}$ & $14 / 21(66,7 \%)$ & $20 / 28(71,4 \%)$ & $34 / 49(69,4 \%)$ & $25 / 38(65,8 \%)$ & $40 / 46(86,9 \%)$ & $65 / 84(77,4 \%)$ \\
\hline $\begin{array}{l}\text { Sollte das Gesundheitsamt an } \\
\text { fehlende Impfungen erinnern? }\end{array}$ & $18 / 20(90,0 \%)$ & $25 / 29(86,2 \%)$ & $43 / 49(87,8 \%)$ & $34 / 36(94,4 \%)$ & $43 / 45(95,5 \%)$ & $77 / 81(95,1 \%)$ \\
\hline
\end{tabular}

a Unterschied zwischen Kinderärzten und Hausärzten signifikant (Impfmeldepflicht bekannt:OR=0,2;95\%-KI:0,1-.0,7; Sind die Eltern mit der namentlichen Impfmeldepflicht einverstanden? OR=0,2; 95\%-KI:0,1-0,6).

Halle war dieser mit 30\% signifikant gröBer als in Magdeburg mit 22,2\%. Die überwiegende Zahl der Mütter, denen die Impfmeldepflicht bekannt war, hatte diese Information vom Kinderarzt erhalten. Ihre $\mathrm{Zu}$ stimmung zur namentlichen Meldepflicht würden 78,2\% der befragten Mütter geben. Noch mehr Mütter, nämlich 83,8\%, würden es begrüßen, wenn das Gesundheitsamt sie an fehlende Impfungen ihrer Kinder erinnern würde. Nicht einmal die Hälfte der Mütter $(44,8 \%)$ war sich dagegen sicher, dass ihr Kinder- oder Hausarzt die namentliche Impfmeldepflicht befürwortet.

Mehr als 95\% der befragten Mütter gaben an, zu vorbeugenden Impfungen ihrer Kinder eine positive bis sehr positive Haltung einzunehmen. Diese Haltung war in Magdeburg etwas ausgeprägter als in Halle. $21,7 \%$ der Mütter lehnten die namentliche Impfmeldepflicht ab. Nach Gründen für diese Ablehnung befragt, wurde hauptsächlich der Datenschutz genannt. Dieser Ablehnungsgrund wurde in Halle häufiger vertreten als in Magdeburg.
- Tabelle 2 zeigt Faktoren, die Einfluss auf die Zustimmung zur Impfmeldepflicht haben könnten. Positiv mit der Zustimmung zur namentlichen Meldepflicht assoziiert waren eine sehr positive bzw. positive Einstellung zum Impfen sowie die Einschätzung, dass der Kinder- und Hausarzt die namentliche Meldepflicht befürwortet. Die Angaben „Impfmeldepflicht bekannt“ bzw. „Impfmeldepflicht durch den Kinderarzt bekannt" hatten insgesamt keinen signifikanten Einfluss auf die Zustimmung zur Impfmeldepflicht. Eine getrennte Betrachtung zwischen Magdeburg und Halle zeigte jedoch, dass diese Einflüsse in Halle sehr wohl nachweisbar waren $(\mathrm{OR}=1,8$; 95\%-KI: 1,1-2,9), nicht aber in Magdeburg $(\mathrm{OR}=0,9 ; 95 \%-\mathrm{KI}: 0,6-1,5)$.

\section{Befragung niedergelassener \\ Kinder- und Hausärzte zur Impfmeldepflicht}

Insgesamt nahmen 50 Kinderärzte ( $\mathrm{Re}$ sponse $82,0 \%$ ) und 211 Hausärzte ( $R e$ - sponse 69,2\%) an der Befragung teil. Die Response der Kinderärzte war in Halle mit 90,6\% höher als in Magdeburg mit 72,4\%. Bei den Hausärzten gab es zwischen Magdeburg und Halle kaum Unterschiede (Magdeburg: 69,4\%, Halle: 68,9\%).

\section{Befragung der Kinderärzte}

Die Ergebnisse der Befragung der Kinderärzte sind in $\bullet$ Tabelle 3 dargestellt. Etwa 90\% der Kinderärzte wussten, dass es eine Impfmeldepflicht gibt. Jedoch gab es aus ihrer Sicht zahlreiche Gründe gegen die Impfmeldepflicht: Mehr als zwei Drittel der Kinderärzte waren der Auffassung, dass die Impfmeldepflicht rechtlich bzw. datenschutzrechtlich unzureichend abgesichert sei. Ein Drittel der Ärzte glaubte, dass die Impfmeldepflicht einen zu hohen Zeitaufwand erfordere. Dagegen waren mehr als 70\% der Kinderärzte der Meinung, dass die Eltern einer namentlichen Meldung zustimmen würden. Die Vorbehalte gegen die Impfmeldepflicht waren bei den Magdeburger Kinderärzten deut- 
lich stärker ausgeprägt als bei den Kinderärzten in Halle.

\section{Die Impferinnerung durch das Gesundheitsamt wird von den befragten Kinderärzten überwiegend für wünschens- wert gehalten}

Die Vorteile der namentlichen Impfmeldepflicht sahen die Kinderärzte beider Städte am ehesten in der Erfassung von Kindern, die keinem Arzt vorgestellt werden und in der nachfolgenden Aufforderung ihrer Eltern, doch noch einen Arzt aufzusuchen, um ihre Kinder impfen zu lassen. Insbesondere in Halle spielte darüber hinaus das Erkennen von Impflücken eine große Rolle. Die Impferinnerung durch das Gesundheitsamt wurde von den Kinderärzten beider Städte ganz überwiegend für sinnvoll und wünschenswert gehalten. Sowohl in Magdeburg als auch in Halle glaubten die befragten Kinderärzte, dass etwa die Hälfte ihrer Berufskollegen der namentlichen Impfmeldepflicht nachkommt.

\section{Befragung der Hausärzte}

Die Ergebnisse der Befragung der Hausärzte sind ebenfalls in $\bullet$ Tabelle 3 dargestellt. Sie erfolgte in gleicher Weise wie die Befragung der Kinderärzte. Zusätzlich wurden die Hausärzte jedoch gefragt, ob sie in ihrer Praxis Kinder bis zum 7. Lebensjahr impfen. Dies beantworteten in Magdeburg 38 von $100(38,0 \%)$ und in Halle 46 von 111 Ärzten $(41,4 \%)$ mit ,ja“. Nur diese Ärzte wurden in die weitere Auswertung einbezogen. Im Gegensatz zu den Kinderärzten war die Impfmeldepflicht unter den Hausärzten weniger bekannt. Die Bedenken hinsichtlich der rechtlichen und datenschutzrechtlichen Absicherung spielten auch bei den Hausärzten eine bedeutende Rolle, jedoch waren sie bei ihnen weniger stark ausgeprägt als bei den Kinderärzten (und in Magdeburg geringer als in Halle). Hingegen war gerade unter den Magdeburger Hausärzten die Auffassung verbreitet, dass die Impfmeldepflicht einen zu hohen Zeitaufwand bedeutet und die Eltern einer Impfmeldepflicht nicht zustimmen würden. Sehr positiv sahen die Hausärzte, insbesondere in Halle, die Vorteile der Impfmeldepflicht für das Erkennen von Impf-

Tabelle 4

Ergebnisse der Befragung der Gesundheitsämter zur Impfmeldepflicht

\begin{tabular}{|c|c|}
\hline Fragebogenangabe & $\begin{array}{l}\text { Ergebnis } \\
\text { Antworten mit ja }\end{array}$ \\
\hline Gibt es im Gesundheitsamt ein altes Impfregister aus der DDR-Zeit? & $24(100,0 \%)$ \\
\hline Wurde das Impfregister nach 1990 genutzt? & $24(100,0 \%)$ \\
\hline \multicolumn{2}{|l|}{ Wofür wurde es genutzt? } \\
\hline - Nachtrag von Impfungen & $24(100,0 \%)$ \\
\hline - Kontrolle des Impfstatus bei Erkrankungshäufungen & $15(62,5 \%)$ \\
\hline - Sonstige Zwecke & $18(75,0 \%)$ \\
\hline $\begin{array}{l}\text { Erhält das Gesundheitsamt Impfmeldungen von niedergelassenen } \\
\text { Ärzten entsprechend § } 4 \text { GDG LSA? }\end{array}$ & $24(100,0 \%)$ \\
\hline \multicolumn{2}{|l|}{ Was passiert mit den Daten im Gesundheitsamt? } \\
\hline - Anlegen eines Impfregisters & $20(83,3 \%)$ \\
\hline -Schriftliche Impferinnerung & $0(0,0 \%)$ \\
\hline \multicolumn{2}{|c|}{ Welche Gründe könnten die Ärzte von ihrer Meldepflicht am ehesten abhalten? } \\
\hline - Keine ausreichende rechtliche Absicherung & $6(25,0 \%)$ \\
\hline - Datenschutz & $7(29,2 \%)$ \\
\hline • Keine Zeit & $18(75,0 \%)$ \\
\hline - Eltern sind dagegen & $5(20,8 \%)$ \\
\hline \multicolumn{2}{|l|}{ Wie könnten die Ärzte motiviert werden, besser zu melden? } \\
\hline - Rückinformation über die Ergebnisse der Impfmeldungen an die & $13(54,2 \%)$ \\
\hline Meldenden & $16(66,7 \%)$ \\
\hline - Erinnerung der Eltern bei fehlenden Impfungen & \\
\hline
\end{tabular}

lücken und die Möglichkeit der Impferinnerung. Sowohl in Magdeburg als auch in Halle vermuteten die befragten Hausärzte, dass etwa 30\% ihrer Berufskollegen der Impfmeldepflicht nachkommen.

\section{Befragung der Gesundheitsämter zur aktuellen Umsetzung der Impfmeldepflicht}

Alle Gesundheitsämter der 21 Landkreise und 3 kreisfreien Städte nahmen an der Befragung teil (Response 100\%). Die wesentlichen Ergebnisse der Befragung sind in - Tabelle 4 dargestellt. Sie zeigen, dass es in allen Gesundheitsämtern ein Impfregister aus der DDR-Zeit gibt. Erfasst wurden hier bis 1990: Name, Vorname, Anschrift, Geburtsdatum, Datum der Impfung, Art und Menge des Impfstoffes, Impfstoffhersteller und Impfstoffcharge [8]. Für die Geburtsjahrgänge 1900-1960 wurde die Tetanusimpfung erfasst. An die alle 10 Jahre anstehende Auffrischimpfung gegen Tetanus wurde schriftlich erinnert. Ab den Geburtsjahrgängen 1960-1989 wurden alle Impfungen (außer BCG-Impfung) entsprechend dem damals geltenden Impfkalender erfasst. Obwohl zwischen der alten und der neuen Meldepflicht etwa 8 Jahre und ein gravierender Strukturwandel im Gesundheitswesen lagen, wurden die Impfregister auch nach 1990 in allen Gesundheitsämtern weiter genutzt, insbesondere für den Nachtrag von Impfungen, wenn Impfausweise verloren gegangen waren. Verletzungen, Reiseberatungen, Impfausweiskontrollen in den Schulen, Belehrungen von Beschäftigten im Lebensmittelbereich nach $\$ 43$ IfSG und Erkrankungshäufungen in Kindereinrichtungen (z. B. durch Pertussis) waren unmittelbare Gründe für den Nachtrag verloren gegangener Impfdokumentationen im Gesundheitsamt.

\section{จ Nur wenige Gesundheitsämter glauben, dass rechtliche oder datenschutzrechtliche Proble- me die Ärzte an der Meldung von Impfungen hindern}

Alle Gesundheitsämter erhielten von niedergelassenen Ärzten Impfmeldungen nach $\$ 4$ GDG LSA. Die Akzeptanz der Impfmeldepflicht bei den Kinderärzten wurde auf mehr als $70 \%(20-100 \%)$, bei den Hausärzten auf etwa 30\% (o-80\%) geschätzt. 18 Gesundheitsämter unterhielten 
für diese Meldungen ein elektronisches Impfregister. Dabei handelte es sich überwiegend um das für den Kinder- und Jugendärztlichen Dienst verwendete Modul eines kommerziellen Anbieters in unterschiedlichen Versionen. Eine aktive schriftliche Impferinnerung für Kinder mit fehlenden Impfungen erfolgte in den Gesundheitsämtern erst im Zusammenhang mit den Impfausweiskontrollen während der Einschulungsuntersuchungen, also nicht im Zusammenhang mit der Impfmeldepflicht. Dies ist mit den bestehenden elektronischen Impfregistern technisch auch nicht möglich.

Als Hinderungsgrund für die Ärzte, ihrer Meldepflicht nachzukommen, vermuteten die Gesundheitsämter den vermeintlich zu hohen Zeitaufwand. Nur wenige Gesundheitsämter glaubten, dass rechtliche oder datenschutzrechtliche Probleme bzw. eine nicht vorhandene Bereitschaft der Eltern die Ärzte an der Meldung hindern. Die Rückmeldung der aufbereiteten Daten an die Ärzte wurde nur von der Hälfte der Gesundheitsämter als motivationssteigernd angesehen. Zwei Drittel der Gesundheitsämter sahen dagegen in einer Impferinnerung eine gute Möglichkeit, die Akzeptanz für die Impfmeldepflicht zu steigern.

\section{Situation in Halle}

In Halle existiert ein Impfregister aus der DDR-Zeit, das für Anfragen niedergelassener Ärzte und Patienten zum Impfstatus sowie für die Erfassung der Impfinanspruchnahme in der Stadt genutzt wird. Es wurde dort geschätzt, dass mehr als 90\% der Kinderärzte, aber nur 2 von mindestens 46 impfenden Hausärzten, ihrer Impfmeldepflicht nachkommen. Mit den neu eingehenden Meldungen wurde versucht, das alte Impfregister fortzuführen. Das Gesundheitsamt bemühte sich durch telefonische und persönliche Kontakte mit den Kinderärzten sehr um die Umsetzung der Impfmeldepflicht nach GDG LSA $\$ 4$.

Im März 2004 wurde in Halle mit der Umstellung auf das elektronische Impfregister begonnen. Erfasst werden mit dieser Software: Namen und Anschriften der meldenden Ärzte, Namen und Anschriften der geimpften Kinder, Geburtstag, Datum der Impfung, Impfstoff bzw. Impfantigene. Die
Berechnung des altersgerechten Impfstatus erfolgt durch die Bewertung der Impfdaten auf der Grundlage des Impfkalenders der Ständigen Impfkommission (STIKO) für Säuglinge, Kinder, Jugendliche und Erwachsene [9]. Es ist möglich, termingemäße Impfungen und fehlende Impfungen zu erkennen sowie die Reihenfolge der Impfungen abzufragen. Die zur Bewertung des Impfstatus realisierten Algorithmen müssen allerdings noch erprobt werden. Mit der Umstellung auf das elektronische Impfregister war zunächst ein Einbruch in der Zahl der meldenden Kinderärzte verbunden. Die diesbezüglichen Ursachen sind unbekannt, es ist aber nicht auszuschließen, dass die aus betrieblich-organisatorischen Gründen stark eingeschränkte Kommunikation des $\mathrm{Ge}$ sundheitsamtes mit den Ärzten nicht unerheblich zu dieser Situation beigetragen haben könnte. Parallel zum Aufbau des elektronischen Impfregisters bemüht sich das Gesundheitsamt gegenwärtig, die Kinderärzte erneut zur Meldung zu motivieren. Die Motivation der Hausärzte zur Meldung ist ein weiteres aktuelles Anliegen des Gesundheitsamtes.

\section{Situation in Magdeburg}

In Magdeburg liegt ein Impfregister aus der DDR-Zeit vor, das für Anfragen niedergelassener Ärzte und Patienten zum Impfstatus genutzt wird. Hier wurde geschätzt, dass etwa $20 \%$ der Kinderärzte namentlich und $10 \%$ nichtnamentlich melden. Bei den Hausärzten liegt die Zahl der namentlich und nichtnamentlich Meldenden bei jeweils 10\%. Das Gesundheitsamt hat die Erfahrung gemacht, dass die Ärzte gravierende rechtliche Probleme bei der Umsetzung der namentlichen Meldepflicht sehen und zudem der Meinung sind, dass die Eltern mit einer namentlichen Meldung nicht einverstanden sind. Allerdings räumt das $\mathrm{Ge}$ sundheitsamt ein, dass den Ärzten Sinn und Zweck der Meldepflicht bisher noch nicht ausreichend vermittelt wurde. Seit 1998 wurde in Magdeburg eine im Gesundheitsamt programmierte dBase-Datenbank als Impfregister verwendet. Die Daten wurden jedoch nur sporadisch eingegeben, da die geringe Zahl meldender Ärzte Zweifel am Sinn des Registers aufkommen ließ. Die Zahl der meldenden Ärzte stieg seit ihrer Befragung zur Impfmeldepflicht zu Beginn des Jahres 2004 deutlich an: Gegenwärtig melden mehr als ein Drittel der Kinderärzte und einige Hausärzte. Inzwischen wurden in Magdeburg neue technische Voraussetzungen für den Aufbau des Impfregisters geschaffen.

\section{Diskussion und \\ Schlussfolgerungen}

Die Bedeutung von Impfregistern für die Erhöhung und Stabilisierung von Durchimpfungsraten zur Zurückdrängung impfpräventabler Krankheiten im Sinne der WHO-Ziele ist international anerkannt. In Australien, Kanada und den USA werden nationale Impfregister oder Impfregisternetzwerke für Kinder bis zum 6. bzw. 7. Lebensjahr aufgebaut. In Australien erfolgt der Aufbau eines nationalen Impfregisters seit 1997. Dort werden die Durchführung von Impfungen und die entsprechenden Meldungen durch die Allgemeinmediziner, die etwa 93\% der australischen Kinder bis zum 7. Lebensjahr mehrmals im Jahr in ihrer Praxis betreuen, durch finanzielle Anreize gefördert. Die Eltern können sich jederzeit mittels eines geschützten Zugangs im Internet oder telefonisch über den Impfstatus ihres Kindes und über anstehende Impfungen informieren [10].

\section{(7) Die Bedeutung von Impfregis- tern für die Zurückdrängung von impfpräventablen Krankhei- ten ist international anerkannt}

In Kanada wird ein Impfregisternetzwerk aufgebaut. Hier liegt die Hoheit für die einzelnen Impfregister in der Hand der Provinzen. Das Netzwerk bildet die Grundlage für ein Impferinnerungssystem. Die Eltern können sich entscheiden, ob sie an fehlende Impfungen erinnert werden möchten. Fachliche Standards und die Kompatibilität der Systeme ermöglichen eine $\mathrm{Zu}-$ sammenführung der Daten auf nationaler Ebene [11]. In den USA wurde ebenfalls mit dem Aufbau eines Impfregisternetzwerkes begonnen, um die bereits erreichten hohen Durchimpfungsraten bei Kindern zu stabilisieren. Ziel ist es dort, bis 2010 95\% aller Kinder unter 6 Jahren zu erfassen. Im Jahr 2002 beteiligten sich bereits 48 der 51 Bundesstaaten am Aufbau eines Impfregisters [12]. In Europa werden in Flandern (Belgi- 
en) sowie in Norwegen Impfregister implementiert: In Flandern handelt es sich um eine Datenbank für Schutzimpfungen bei Kindern [13], in Norwegen werden ca. 70\% aller Schutzimpfungen in einem zentralen nationalen Impfregister erfasst [14].

In Deutschland besteht nur in SachsenAnhalt eine namentliche Impfmeldepflicht. In Mecklenburg-Vorpommern regelt eine Landesverordnung von 1994 die anonyme Meldung von Impfungen ab dem Geburtsjahrgang 1976 [15]. Die nichtnamentliche Meldung ermöglicht zwar eine Schätzung der Impfinanspruchnahme, jedoch nicht die Beurteilung des altersgerechten Impfstatus eines Jahrgangs und auch nicht die Erinnerung an fehlende Impfungen. Die Sächsische Impfkommission (SIKO) empfiehlt seit 2003 die namentliche Meldung aller Impfungen an das Gesundheitsamt. Für diese Meldungen ist das schriftliche Einverständnis des Impflings oder seines Sorgeberechtigten erforderlich [16]. Deutschlandweit ist lediglich die Registrierung der Impfdaten einzuschulender Kinder geregelt. Rahmenbedingungen für die Einführung eines nationalen Impfregisters oder Impfregisternetzwerkes wie in den USA und Kanada gibt es nicht.

Sachsen-Anhalt hat mit der Regelung der Impfmeldepflicht in Deutschland eine bemerkenswerte Vorreiterrolle übernommen. Schwierigkeiten bei der Umsetzung der Meldepflicht sollten insbesondere mit der Befragung junger Mütter und niedergelassener Ärzte analysiert werden. Die Rückmeldung (Response) auf die Befragung der Mütter entsprach mit knapp 54\% den Erwartungen an eine postalische Befragung mit einmaliger Erinnerung [7]. Die Response der Befragung der Hausärzte und der Kinderärzte war mit knapp 70\% bzw. 82\% erfreulich hoch. Allerdings ist nicht auszuschließen, dass eher Mütter bzw. Ärzte an der Befragung teilnahmen, die dem Impfen und der Impfmeldepflicht positiv gegenüberstehen. Es zeigte sich, dass die jungen Mütter in Halle und Magdeburg ihre Zustimmung zur Impfmeldepflicht stark von der entsprechenden Haltung des impfenden Arztes abhängig machen. Die impfenden Ärzte, insbesondere die Kinderärzte, haben jedoch in Bezug auf die rechtliche bzw. datenschutzrechtliche Absicherung der Impfmeldepflicht Vorbehalte. Es besteht Handlungsbedarf, um diese Beden- ken auszuräumen. Die Hausärzte befürchten zudem einen zu großen Zeitaufwand. Hier ist die Unterstützung durch das Gesundheitsamt über die Bereitstellung vorgefertigter Meldelisten, Einverständniserklärungen und Informationsmaterialien für die Eltern erforderlich.

Die Impferinnerung durch das Gesundheitsamt wird von fast $84 \%$ der befragten jungen Mütter befürwortet. Eine diesbezüglich sinnvolle und effektive Verfahrensweise soll im Zusammenhang mit dem Aufbau elektronischer Impfregister und in $\mathrm{Ab}$ stimmung mit den meldenden Ärzten erarbeitet und getestet werden. Eine ständige Kommunikation zwischen dem Gesundheitsamt und den niedergelassenen Ärzten sowie die Erfahrung, dass das Impfregister auch für die Meldenden unmittelbar von Vorteil ist, kann darüber hinaus das Interesse der Ärzte an der Impfmeldung steigern. Die Unterstützung der Gesundheitsämter beim Aufbau eines an einheitlichen Standards ausgerichteten und technisch kompatiblen Impfregisters ist ebenso unerlässlich wie die Zusammenführung der anonymisierten Daten. Für beide Aufgaben steht das Landesamt für Verbraucherschutz Sachsen-Anhalt als Fachbehörde für den öffentlichen Gesundheitsdienst zur Verfügung. Es ist in der Lage, die Daten analog zu den Impfdaten einzuschulender Kinder in die Landesgesundheitsberichterstattung einfließen zu lassen und diese für die Einschätzung des Stands der Umsetzung des Landesgesundheitsziels zu nutzen. Der Aufbau des Impfregisters in Sachsen-Anhalt als Impfregisternetzwerk mit primärer Ansiedlung in den Gesundheitsämtern erscheint bereits jetzt als Vorteil: Hier können sowohl die Motivation zur Meldung als auch Interventionen mit regionalem Bezug (beispielsweise Impfprojekte bzw. Information und Aufklärung klar definierter Zielgruppen) Erfolg versprechend durchgeführt werden. Zudem zählt die Förderung des Impfgedankens zu den originären Aufgaben der Gesundheitsämter.

\section{Korrespondierender Autor Dr. H. Oppermann}

Fachbereich Gesundheit, Hygiene, Epidemiologie, Landesamt für Verbraucherschutz Sachsen-Anhalt, Wallonerberg 2-3, 39104 Magdeburg E-Mail: Hanna.Oppermann@md.lav.ms.Isa-net.de

\section{Danksagung}

Wir danken allen an der Studie Beteiligten für die Unterstützung und der Firma GlaxoSmithKline für die Förderung.

\section{Literatur}

1. Gesetz über den Öffentlichen Gesundheitsdienst und die Berufsausübung im Gesundheitswesen im Land Sachsen-Anhalt (1997) GVBI. LSA 55:10231030

2. Oppermann H, Kolbe M (2002) Von den Mühen der Ebene: Gesundheitsziel „Erreichen eines altersgerechten Impfstatus bei über $90 \%$ der Bevölkerung in Sachsen-Anhalt". Gesundheitswesen 64:664668

3. Hens C (1998) Impfmeldung an die Gesundheitsämter. Pro 11:382

4. Kunze MT, Sroka S (2000) Meldepflicht für Schutzimpfungen bei Kindern bis zum vollendeten 7. Lebensjahr. Pro 4:157

5. Kudela G (2001) Aufbau eines altersgerechten Impfschutzes bei unseren Kindern. Pro 6:189

6. Jungbauer-Gans M, Kriwy P (2003) Der Arzteinfluss auf die Durchimpfungsrate. Gesundheitswesen 65:464-470

7. Reuband KH (2001) Möglichkeiten und Probleme des Einsatzes postalischer Befragungen. Kölner Z Soziologie Sozialpsychologie 53:307-333

8. Dittmann S, Thilo W (1980) Die Organisation des Impfwesens in der DDR. In: Vademekum für Impfärzte. Gustav Fischer, Jena, S 33-44

9. Empfehlungen der Ständigen Impfkommission (STIKO) am Robert Koch-Institut (2003) Epidemiologisches Bulletin des Robert Koch-Instituts 32:246

10. Crampton RM (1995) The Australian childhood immunization register (ACIR). Aust Fam Physician 24:1392-1393

11. Functional standards and minimum (core) data sets for a national Immunization Registry Network and vaccine Associated Adverse Events Surveillance system (VAAESS) (2002) Can Commun Dis Rep 28:1-32

12. Initiative on Immunization Registries (2001) MMWR, Recommandations and Reports 50:1-17

13. Dewolf $D(2000)$ Vaccination policy in Flanders. Verh K Acad Geneeskd Belg 62:269-280

14. Ree $A O$ (1993) Vaccination coverage in local and national perspective. Vaccination coverage before and after introduction of a local computerized system in the municipality of Sandefjord. Tidsskr Nor Laegeforen 113:225-229

15. Verordnung über die Meldedaten von Impfungen und atypischen Impfverläufen (Impf-Melde-Verordnung - ImpfMVO) (1994), GVBI. Meckl.-Vorp. 26:1083

16. Empfehlungen der Sächsischen Impfkommission zur Organisation der Dokumentation von Schutzimpfungen (2003) LUA-Mitteilungen 11:18-19 